\title{
La psiquiatrización de la vida cotidiana: el DSM y sus dificultades*
}

Psychiatrization of Normal Life: DSM and Its Troubles

Sandra Caponi ${ }^{\dagger}$

\begin{abstract}
Resumen
En los días que se siguieron a la publicación de la quinta edición del Manual de Diagnóstico y Estadística de Enfermedades Mentales (DSM-5), diversas voces se levantaron contra esa nueva clasificación diagnóstica, pues había aumentado la sensación, ya dejada por las anteriores ediciones, de ser una clasificación epistemologicamente poco consistente. Entre estas voces dos críticas concentraron la atención de la comunidad internacional, ambas realizadas por psiquiatras americanos: la crítica realizada por Thomas Insel, director del National Institute of Mental Health (NIMH) y la crítica realizada por Allen Frances, jefe del grupo de tareas del equipo que elaboró el DSM-IV. Considerando el impacto de esas dos críticas, pretendo analizar aquí los límites y dificultades que cada de ellas presenta. Aunque ambos critican el Manual, sus argumentos terminan por reforzar un retorno a la psiquiatría biológica y a la anticipación de riesgos, desconsiderando las reales dificultades implícitas en la propuesta clasificatoria del DSM-5.
\end{abstract}

Palabras clave: DSM-5 - riesgo - clasificación - psiquiatría - enfermedades mentales

\begin{abstract}
In the days after the publication of the fifth edition of the Diagnostic and Statistical Manual of Mental Disorders (DSM-5), many voices were raised against this new diagnostic classification, increasing the sensation left by the previous editions, of it being a classification of low epistemological consistency. Among these voices, two criticisms attracted the attention of the international community, both from American psychiatrists: the critique made by Thomas Insel, director of the National Institute of Mental Health (NIMH); and the critique made by Allen Frances, chief of the task force team that produced the DSM-IV. Considering the impact of both criticisms, here I discuss the limits and difficulties that each of them presents. Although both authors criticize the Manual, their arguments reinforce a return to biological psychiatry and the anticipation of risks, disregarding the real difficulties involved in the classification proposed by the DSM-5.
\end{abstract}

Keywords: DSM-5 - risk - classification - psychiatry - mental illness

\footnotetext{
* Recibido: 7 de Marzo de 2016. Aceptado con revisiones: 5 de Diciembre de 2016.

† Universidade Federal de Santa Catarina/CNPq. Para contactar al autor, por favor, escribir a: sandracaponi@gmail.com. Metatheoria 8(2)(2018): 97-103. ISSN 1853-2322.

(c) Editorial de la Universidad Nacional de Tres de Febrero. Publicado en la República Argentina.
} 


\section{Introducción}

El día 22 de mayo de 2013 la Asociación de Psiquiatría Americana (APA) divulgó la finalización de la quinta edición del Manual de Diagnóstico y Estadística de Enfermedades Mentales (APA 2013), mundialmente conocido como DSM-5. Ese grupo estaba encargado de revisar y discutir la clasificación psiquiátrica existente, utilizada hasta ese momento como referencia en el mundo entero, el DSM IVTR. Sin embargo, el proceso de recepción de esta nueva versión del DSM no fue tan tranquilo como se esperaba.

En los días que se siguieron a la publicación de la quinta edición, diversos autores mostraron su oposición ${ }^{1}$ a esa nueva clasificación diagnóstica, por haber aumentado una sensación, ya dejada por las anteriores ediciones del Manual. El DSM-5, como sus antecesores, presentaba una clasificación de enfermedades mentales epistemológicamente poco consistente, con una fuerte tendencia a multiplicar innecesariamente los diagnósticos, abriendo la puerta para la creación e invención de nuevas patologías psiquiátricas.

El DSM-IV editado en el año 1994 ya había multiplicado el número de diagnósticos psiquiátricos centrándose en la identificación de síntomas clínicos bastantes ambiguos, posibilitando, de ese modo, el surgimiento de nuevas terapéuticas, generalmente farmacológicas. La existencia inevitable de fronteras inestables, difusas y ambiguas entre el normal y el patológico en el campo de la salud mental parece haber posibilitado el creciente proceso de medicalización de conductas, que pasaron a ser consideradas como socialmente indeseables y a ser clasificadas como anormales. Asistimos, así, a la creciente consolidación de ese espacio de saber y de intervención que Michel Foucault (1999) denominó con el nombre de medicina de lo no patológico.

El DSM-5 se inscribe en esa misma estrategia que caracterizó al Manual de Diagnóstico y Estadística de Enfermedades Mentales (APA 1980), el DSM-III, desde la ruptura que este Manual produjo en el campo de la psiquiatría, en el año 1980. A partir de ese momento las patologías psiquiátricas pasaron a ser clasificadas teniendo como referencia nuevas agrupaciones de síntomas. Desde entonces, lo que llevó a desconsiderar (o a dejar en segundo plano) las narrativas de los pacientes.

\section{Clasificar los sufrimientos}

Clasificar significa agrupar de acuerdo a un criterio pre-establecido, ordenar un conjunto complejo de datos. Para eso, resulta necesario identificar las individualidades, las características comunes a un determinado grupo que permita legitimar la creación de conjuntos. Para poder clasificar algo es necesario conocer el universo al cual ese algo pertenece, esto es, definir la totalidad de la cual forma parte.

Es por eso que la clasificación de patologías generales solo pudo transformarse en un instrumento de uso generalizado cuando las particularidades de las enfermedades orgánicas fueron científicamente y socialmente aceptadas por el saber médico, un fenómeno que se consolidó en la segunda mitad del siglo XIX. Solo cuando la medicina general fue capaz de establecer correlaciones entre conjuntos de síntomas y lesiones orgánicas específicas, fue posible crear una clasificación de enfermedades con pretensiones de universalidad. Inicialmente surgió la "Clasificación Bertillon", en el año 1893, y más tarde la Clasificación Internacional de Enfermedades (CIE) establecida por la OMS.

Y es justamente en ese contexto que se sitúan las críticas dirigidas a la clasificación de patologías psiquiátricas. El esfuerzo por crear una clasificación universal de patologías mentales tiene su inicio en el año 1889, cuando se organizó en París la Primera Clasificación Internacional de Enfermedades Mentales. Esa ambición clasificatoria se consolida en 1952, con la publicación del DSM-1, manteniéndose

\footnotetext{
${ }^{1}$ Análisis críticos sobre esa clasificación fueron realizados por autores como Berrios (2008), Roudinesco (2013), Paris y Phillips (2013), Sadler (2010, 2013), Horwitz (2013), Horwitz y Wakefield (2007), Conrad (2007), Braunstein (2013), Coliman (2009), Ribeiro Guedes, Nogueira y Camargo (2006), entre otros.
} 
inalterada en las diferentes versiones posteriores del DSM. Sin embargo, el campo de la psiquiatría nunca llegó a conquistar el objetivo de establecer, como lo requiere el modelo explicativo de la medicina general, para cada conjunto de síntomas una alteración neuroquímica o neurológica específica.

La publicación de DSM-5 suscitó diversas críticas que se centraron en la fragilidad epistemológica que históricamente caracterizó a ese Manual, una fragilidad que parecería haberse agravado, aún más, en esta versión. Entre los debates suscitados a propósito del DSM-5, la crítica que tuvo mayor impacto fue a realizada por Thomas Insel, cuando, poco antes de la publicación de la quinta versión del Manual, (Insel 2013) anunciaba que el National Institute of Mental Health (NIMH) abandonaría la utilización de la clasificación propuesta por la APA, por tratarse de una lista de síntomas sin una base científica sólida. Esa declaración parecía indicar el fin de la hegemonía clasificatoria del DSM. Sin embargo, una lectura atenta de la posición defendida por Insel resulta aún más perturbadora que la propia reedición del DSM.

Esa crítica, en verdad, nada agrega al debate. Al contrario, retoma los postulados localizacionistas que caracterizaron a la psiquiatría biológica del siglo XIX. En cada esfuerzo por encontrar la localización cerebral, o la explicación neuroquímica para trastornos mentales, la respuesta parecía ser la misma: aún no fue posible alcanzar los resultados esperados, pero un futuro promisorio se anuncia para la explicación biológica de patologías mentales. Vemos repetirse el mismo argumento desde hace más de cien años, cuando se enuncia la convicción de que, en un futuro próximo, serán definidas alteraciones cerebrales específicas para cada una de nuestras aflicciones.

A diferencia de patologías como SIDA o la enfermedad de Chagas, no existen marcadores biológicos definidos, sino solo suposiciones y convenciones. Por esa razón Insel pudo afirmar que "La debilidad del DSM está dada por su falta de validez" (Insel 2013, p. 2), esto es, por su falta de referencia a estudios de laboratorio capaces de validar los diagnósticos. Ningún marcador biológico impide que esas clasificaciones sean alteradas y modificadas a través de acuerdos y negociaciones, pues ellas son como afirmaría Desrosiéres (2010), “convenciones colectivas de equivalencia”. Son conjuntos definidos por votación, después de largas negociaciones y discusiones, por los miembros de la Asociación de Psiquiatras Americanos (APA). El objetivo de ese proceso de discusión y votación de la APA no es otro que crear un lenguaje común, universal, a partir del cual se puedan establecer procesos de medición y comparación.

Es preciso formular una pregunta frente a la crítica formulada por Thomas Insel referida a la ausencia de una base científica del DSM: iserá qué Insel propone sustituir una arbitraria y convencional estrategia de agrupación de síntomas, por la escucha atenta a la narrativa de los pacientes? Ciertamente no. Lo qué Insel anhela es el regreso a los sueños fracasados del siglo XIX, quiere que se logre, por fin, la ansiada identificación de la psiquiatría con los procedimientos utilizados por la medicina general.

Imaginar que nuestros sufrimientos psíquicos pueden ser tratados como una úlcera o una infección significa simplemente negar que cada sufrimiento se inscribe en una historia de vida. Significa ignorar que nuestros padecimientos se transforman a lo largo de nuestras vidas, y que esa transformación no ocurre independientemente del modo como somos escuchados, ni independientemente de las intervenciones o terapéuticas que nos han propuesto.

La segunda crítica es inseparable de la anterior. Pues, si no existe un marcador biológico que permita validar las agrupaciones de síntomas, pueden ser realizadas infinitas combinaciones posibles, esto es, podrán surgir siempre nuevos y nuevos diagnósticos. Esa elasticidad de las clasificaciones fue problematizada por el psiquiatra Allen Frances, jefe del grupo de tareas de la APA que organizó el DSM-IV.

Frances considera que, dada la dificultad existente para establecer límites precisos entre normalidad y patología psiquiátrica, la clasificación de diagnósticos para trastornos mentales puede ser indefinidamente ampliada, apareciendo a cada nueva edición, nuevos diagnósticos y reagrupaciones cada vez más amplias como es el caso de los "trastornos de espectro autista". 
Las críticas de Allen Frances al DSM-5 empezaron a ganar difusión en el año 2010 con la publicación de su artículo "Opening Pandora's Box: The 19 Worst Suggestions for DSM5" (Frances 2010), cuando aún el Manual se encontraba en proceso de discusión y elaboración. Más tarde esas críticas se consolidaron en el libro Saving Normal: An Insider's Revolt Against Out-of-Control Psychiatric Diagnosis, DSM-5, Big Pharma, and the Medicalization of Ordinary Life (Frances 2013a).

\section{Anticipar los riesgos}

Frances ya había manifestado anteriormente su preocupación por la categoría Psychosis Risk Syndrome. Esta categoría fue excluida del DSM-5, pero reapareció con otro nombre, ahora recibiendo la denominación de Attenuated Psychosis Syndrome, incluida dentro del grupo Schizoprenia Spectrum. Frances dirá que:

La única manera de evitar los peligros del DSM-5 es estar plenamente conscientes de ellos. No tiene absolutamente ningún sentido fijar el rótulo engañoso y estigmatizante "Other Specified Schizophrenia Spectrum Disorder" en alguien que, en configuraciones típicas, tendrá apenas cerca del 10\% de chance de volverse psicótico. Y, ciertamente, no tiene sentido seguir ese diagnóstico equivocado con tratamientos antipsicóticos sin comprobación y potencialmente muy perjudiciales. (Frances 2013b, p. 1)

Sin embargo, ésa no es una característica exclusiva del DSM-5, como parece sostener Frances, pues la obsesión por anticipar y prevenir riesgos ya estaba presente en las anteriores ediciones del DSM. Por ejemplo, cuando se afirmaba que la existencia de uno o más síntomas (de los nueve definidos en el DSM-IV) para depresión ya indicaría la existencia de riesgo de padecer un episodio depresivo en el futuro.

En diversos textos, y dando continuidad a lo que afirmara en "Opening Pandora's Box" (2010), Frances dirá que el del DSM-5 anuncia la aparición de una verdadera pandemia de trastornos mentales (Frances 2010, 2012, 2013). Para Frances: "El DSM-5 podría crear decenas de millones de nuevos pacientes 'falsos positivos', exacerbando así los problemas causados por un ya inclusivo DSM-IV. Habría excesivos tratamientos masivos con medicaciones innecesarias, de alto costo y frecuentemente bastantes perjudiciales" (Frances 2010, p. 2).

El hecho de que esas propuestas lleven a clasificar inadecuadamente con el rótulo de "trastorno mental" a millones de personas que antes eran consideradas "normales" no es nuevo, ni accidental, ni resulta de una elección metodológica equivocada del Grupo de Tareas del DSM-5. Se trata, al contrario, de la aplicación de una estrategia teórica y política que se articula en torno a la idea de riesgo. Una estrategia contemporánea a la propia ambición clasificatoria de la psiquiatría, en la que Frances tuvo un papel central.

De acuerdo con esa estrategia utilizada por los psiquiatras de la APA, el incremento de la tasa de trastornos mentales en la población ocurre de dos modos. En primer lugar, este aumento está vinculado a la aparición de nuevos diagnósticos que transforman en patológicos comportamientos comunes en la sociedad, conductas que la industria farmacéutica se encarga de difundir y popularizar (como la tristeza, los pequeños déficit de cognición, las explosiones de rabia, los comportamientos sexuales, las adicciones a ciertas conductas cotidianas como comprar, el uso de sustancias tóxicas, etc.). En segundo lugar, se aumentarán los trastornos estableciendo un umbral de diagnóstico más bajo para muchas patologías ya existentes, como ocurrió al retirar a excepcionalidad concedida a los casos de duelo para el diagnóstico de depresión (Frances 2010, 2013). Así, mientras en el DSM-IV el luto era considerado una situación excepcional, aceptándose la permanencia de síntomas indeseados (por ejemplo: alteración de sueño y apetito, sentimiento de inutilidad o culpa) por dos meses, en el DSM-5 esa excepcionalidad desaparece (APA 2013, p. 162). Se considera que después de un duelo, independientemente de tratarse o no de la muerte de un ser querido próximo, los síntomas deberán desaparecer en dos semanas, de lo contrario, esa persona que se encuentra atravesando por la inevitable tristeza de la pérdida recibirá un diagnóstico de depresión. 
Es posible afirmar que una de las estrategias indispensables para garantizar la indefinida ampliación de diagnósticos y categorías psiquiátricas es la obsesión por identificar pequeñas anomalías, sufrimientos cotidianos, pequeños desvíos de conducta como indicadores de una patología psiquiátrica grave por venir. El riesgo, en la medida en que aparece como un modo de anticipar un peligro posible (real o imaginado) sobre la vida y la salud, constituye la estrategia privilegiada que permite garantizar la legitimidad y aceptabilidad de la multiplicación de diagnósticos. Frances afirma:

Los psiquiatras esperan identificar pacientes más temprano y crear tratamientos efectivos para reducir la cronicidad de las patologías. Desafortunadamente, los miembros del Grupo de Tareas comúnmente cometen el error de olvidar que cualquier esfuerzo por reducir las tasas de falsos negativos debe inevitablemente elevar las tasas de falsos positivos (frecuentemente de modo dramático y con fatales consecuencias). Si alguna vez será posible conseguir la esperada ventaja de detección precoz de casos, deberemos tener pruebas diagnósticas específicas y tratamientos seguros. Al contrario, las propuestas del DSM-5 llevan a la particularmente peligrosa combinación de diagnósticos no específicos e inadecuados, y de tratamientos no probados y perjudiciales (Frances 2010, p. 6).

Podemos afirmar que la problemática del riesgo (Castiel 2007) es la estrategia más utilizada para legitimar la expansión de enfermedades mentales. En ese sentido podemos entender la afirmación de Frances, según la cual, el Síndrome de riesgo de psicosis (posteriormente llamado Attenuated Psychosis Syndrome) provocaría una alarmante tasa de falsos positivos, entre 70 a $75 \%$, llevando a cientos de millares de adolescentes y jóvenes a recibir, sin necesidad, la prescripción de antipsicóticos atípicos que causan efectos colaterales serios tales como aumento de peso, impotencia sexual y reducción de la expectativa de vida. De modo que,

La prevención de la psicosis sería una óptima idea, si realmente fuese posible hacerlo, pero no hay ninguna razón para pensar que podemos. Ir más allá de nuestra comprensión probablemente afectará aquéllos que esperábamos ayudar. El Riesgo de Psicosis no debe ser usado como un diagnóstico clínico, pues será casi siempre equivocado. La ruta para el infierno está adoquinada de buenas intenciones y de malas consecuencias no intencionales. Primero, no causar daño (Frances 2013c, p. 1).

La multiplicación de categorías diagnósticas que aparecen en los sucesivos DSM desde el año 1980, forman parte de esta lógica que promete anticipar el riesgo de sufrir una patología mental grave en el futuro, posibilitando la creciente multiplicación de patologías mentales en la infancia (TDAH, dislexia, ansiedad, entre otras).

Por fin, debemos decir que la estrategia de clasificación de diagnósticos psiquiátricos no puede ser pensada desconsiderando el papel desempeñado por la poderosa industria farmacéutica. Esa relación es, sin embargo, sistemáticamente negada por los psiquiatras, aún por los más críticos. Así, cuando Frances es interrogado por la relación que existe entre los miembros de la APA y la industria farmacéutica, niega cualquier interferencia y formula esta sugestiva pregunta retórica: “¿Cómo pueden personas tan inteligentes y escrupulosos (refiriéndose a sus amigos y colegas del Grupo de Tareas) hacer tantas sugerencias malas?" (Frances 2012, p. 5). La respuesta será:

Ha sido mi experiencia consistente (obtenida trabajando en los tres previos DSMs) que cada grupo de Trabajo tiene siempre una fuerte (frecuentemente irresistible) ansia de expandir los límites de los desórdenes de su sección. Ese previsible imperialismo de diagnóstico de los Grupos de Tarea debe ser siempre reconocido y resistido. Los especialistas tienen mucha expectativa en reducir los falsos negativos para sus trastornos favoritos y en anular la necesidad de recurrir a la etiqueta "no especificado de otro modo" (Frances 2012, p. 5).

Difícilmente podremos llegar a tener un ejemplo más claro del modo como opera la medicalización de comportamientos cotidianos y de sufrimientos psíquicos leves. 


\section{Para concluir}

Sabemos que la atribución de un diagnóstico tomando como referencia una lista de síntomas, muchas veces obscurece y silencia contextos sociales o situaciones de vida que puede ser fatal desconsiderar, tales como la violencia familiar o el asedio moral en el trabajo. La identificación de diagnósticos a partir de un conjunto de síntomas obscurece los conflictos sociales e individuales que pueden haber desencadenado los sufrimientos.

Así, desconsiderando la pluralidad de contextos en los cuales pueden aparecer los sufrimientos, siempre que exista un conjunto determinado de síntomas (alteración de sueño y apetito, sentimiento de infelicidad o culpa, tristeza profunda) se tenderá a realizar un mismo diagnóstico, utilizando un mismo código burocrático y una misma medicación. Eso podrá ocurrir independientemente del hecho de desconocerse tanto los conflictos sociales e individuales, como la etiología biológica y las alteraciones neuroquímicas implicadas. Ese desconocimiento queda explícitamente enunciado, por ejemplo, en la bula de un medicamento cada vez más consumido por niños y jóvenes para tratar una supuesta patología como el TDAH, me refiero a la Ritalina. Podemos leer en el prospecto que acompaña a ese medicamento, particularmente en la rúbrica Consideraciones especiales sobre el diagnóstico de TDAH, la siguiente afirmación: "la etiología específica de ese síndrome es desconocida y no existen pruebas diagnósticas específicas” (Novartis 2013).

Creo que, como afirman Elisabeth Roudinesco (2013) o Phylippe Pignarre (2006), entre otros, resulta necesario abandonar el DSM como modelo hegemónico de diagnóstico en el campo de la psiquiatría. Se trata de un Manual que necesariamente reduce los sufrimientos individuales a una lista de síntomas ambiguos y poco claros para un conjunto, cada vez mayor, de patologías mentales. Es preciso inventar estrategias que nos permitan comprender que los sufrimientos psíquicos solo pueden volverse inteligibles en el interior de una historia de vida. Pues, será solamente la escucha atenta de las narrativas de nuestros odios y amores, de nuestros miedos, conquistas y fracasos lo que nos auxiliará en la difícil e infinita tarea de construcción y reconstrucción de nuestra subjetividad.

Bibliografía

American Psychiatric Association (2013), Diagnostic and Statistical Manual of Mental Disorders, Fifth Edition (DSM-5), Arlington: American Psychiatric Association.

American Psychiatric Association (1980), Diagnostic and Statistical Manual of Mental Disorders, Third Edition (DSM-III), Arlington: American Psychiatric Association.

Braunstein, A. (2013), Clasificar en psiquiatría, México: Siglo XXI.

Berrios, G. (2008), Historia de los Sintomas de los trastornos mentales, México: Fondo de Cultura Económica.

Castiel, L. (2007), A Saúde Persecutória e os limites da responsabilidade, Rio de Janeiro: Fiocruz.

Caliman, L.V. (2009), “A constituição sócio-médica do “fato TDAH”, Psicologia Ė Sociedade 21(1): 135-144.

Ribeiro Guedes, C., Nogueira, M. y K.Jr. Camargo (2006), "A subjetividade como anomalia: contribuições epistemológicas para a crítica do modelo biomédico”, Ciência $\mathcal{E}$ Saúde Coletiva 11(4): 1093-1103.

Conrad, P. (2007), The Medicalization of Society, Baltimore: The Johns Hopkins University Press.

Desrosiéres, A. (2010), La politique des grands nombres: histoire de la raison statistique, Paris: La Découverte.

Foucault, M (1999), Les Anormaux, Paris: Seuil.

Frances, A. (2010), “Opening Pandora's Box: The 19 Worst Suggestions For DSM5”, Review of Psychiatric Times 1(11): $1-10$. 
Frances, A. (2012), “DSM-5 Continues to Ignore Criticism from Petitioners”, Huffingtonpost, 20 de junio. Accesible en: http://www.huffingtonpost.com/allen-frances/DSM-V-petition_b_1610569.html?view=print\&comm_ref=false.

Frances, A. (2013a), Saving Normal: An Insider's Revolt Against Out-of-Control Psychiatric Diagnosis, DSM-5, Big Pharma, and the Medicalization of Ordinary Life, New York: Harper Collins Publisher.

Frances, A. (2013b), "Psychosis Risk Syndrome Is Back to Haunt Us", Huffingtonpost, 26 de noviembre. Fecha de publicación: 20/11/2013. Accesible en: http://www.huffingtonpost.com/allen-frances/psychosis-risk-syndromei_b_4343661.html.

Horwitz, A. (2002), Creating Mental Illness, Chicago: The University of Chicago Press.

Horwitz, A. y J. Wakefield (2007), The Loss of Sadness, Oxford: Oxford University Press.

Insel, T. (2013), “Transforming Diagnosis”, NIMH, 29 de abril. Accesible en: http://www.nimh.nih.gov//about/director/directors-biography.shtml

Paris, J. y J. Phillips (eds.) (2013), Making the DSM-5: Concepts and Controversies, New York: Springer.

Pignarre, P. (2006), Les malheurs des psys: psychotropes et médicalisation du social, Paris: La Découverte.

Roudinesco, E. (2013), ¿Por qué el psicoanálisis?, Buenos Aires: Paidós.

Stop DSM (2013), "To Oppose the DSM-5 is not to Oppose Psychiatry". Accesible en: http://www.stopdsm.org/index.php/en/. Fecha de acceso: 10/12/2013.

Sadler, J. (2010), "Waiting for the Miracle”, Bulletin: Association for the Advancement of Philosophy and Psychiatry 17(1): 14.

Sadler, J. (2013), "Considering the Economy of DSM Alternatives”, en Paris, J. y J. Phillips (eds.), Making the DSM-5: Concepts and Controversies, New York: Springer, pp. 21-38. 